(C) 2020. This manuscript version is made available under the CC-BY-NC-ND 4.0 license http://creativecommons.org/licenses/by-nc-nd/4.0/

The according paper is published by Elsevier under the following link:

https://www.sciencedirect.com/science/article/pii/S1540748918302396?via\%3Dihub

(DOI : 10.1016/j.proci.2018.06.056)

\title{
Ab initio kinetics predictions for H-atom abstraction from diethoxymethane by hydrogen, methyl, and ethyl radicals and the subsequent unimolecular reactions
}

Leif C. Kröger ${ }^{a, *}$, Malte Döntgen ${ }^{a, b}$, Dzmitry Firaha ${ }^{a, c}$, Wassja A. Kopp ${ }^{a}$, Kai Leonhard ${ }^{\mathrm{a}, \mathrm{c}}$

${ }^{a}$ Chair of Technical Thermodynamics, RWTH Aachen University, 52062 Aachen, Germany

${ }^{b}$ Present address: Molecular Science, Department of Chemistry, University of Helsinki, FI-00560 Helsinki, Finland

${ }^{c}$ AICES Graduate School, RWTH Aachen University, 52062 Aachen, Germany

\section{Corresponding author:}

Leif C. Kröger

Chair of Technical Thermodynamics

RWTH Aachen University

52062 Aachen, Germany

Phone: 00492418095356

Mail: leif.kroeger@ltt.rwth-aachen.de

Colloqium topic: Gas-phase reaction kinetics

Total length of paper: 6.75 pages (Method 2)

Supplemental Material: Yes

Color reproduction: no (figures to be printed in gray scale)

\footnotetext{
*Corresponding author:

Email address: leif.kroeger@ltt.rwth-aachen.de (Leif C. Kröger)
} 


\title{
Ab initio kinetics predictions for $\mathrm{H}$-atom abstraction from diethoxymethane by hydrogen, methyl, and ethyl radicals and the subsequent unimolecular reactions
}

\author{
Leif C. Kröger ${ }^{\mathrm{a}, *}$, Malte Döntgen ${ }^{\mathrm{a}, \mathrm{b}}$, Dzmitry Firaha ${ }^{\mathrm{a}, \mathrm{c}}$, Wassja A. Kopp ${ }^{\mathrm{a}}$, Kai Leonhard ${ }^{\mathrm{a}, \mathrm{c}}$ \\ ${ }^{a}$ Chair of Technical Thermodynamics, RWTH Aachen University, 52062 Aachen, Germany \\ ${ }^{b}$ Present address: Molecular Science, Department of Chemistry, University of Helsinki, FI-00560 Helsinki, Finland \\ ${ }^{c}$ AICES Graduate School, RWTH Aachen University, 52062 Aachen, Germany
}

\begin{abstract}
Diethoxymethane (DEM) is a promising oxygenated fuel and fuel additive, which has similar positive combustion characteristics as dimethoxymethane. DEM contains $\mathrm{C}$ - $\mathrm{C}$ bonds and can form ethylene via $\beta$-scission, which potentially increases sooting tendency. Since DEM is rarely studied, however, kinetic modeling attempts are forced to rely on rate constant analogies. Therefore, we employ high level CCSD(T)/aug-cc-pV(T+D)Z//B2PLYPD3BJ/6$311++(\mathrm{d}, \mathrm{p})$ theory along with transition state theory to predict reaction rate constants for $\mathrm{H}$-abstraction by $\dot{\mathrm{H}}$ and $\dot{\mathrm{CH}}_{3}$ and the subsequent unimolecular reactions. We further prove that the DLPNO approximation to $\operatorname{CCSD}(\mathrm{T})$ leads to a deviation of less than $0.25 \mathrm{kcal} / \mathrm{mol}$ in barrier heights for the presently studied open-shell electronic structures and use it for the prediction of reaction rate constants for $\mathrm{H}$-abstraction by $\mathrm{C}_{2} \mathrm{H}_{5}$ radicals. We find that $\mathrm{H}$-abstraction by ethyl radicals might denote a significant pathway, which should not be neglected in kinetic modeling studies of DEM. It is also shown that reaction pathways leading to ethylene formation are of minor importance and give thereby a first insight into the fate of the C-C bonds. To the best of our knowledge, this study represents the first high-level ab-initio study of DEM, which makes the reaction kinetics and thermochemistry data provided by this study vital for future comprehensive kinetic modeling of DEM.
\end{abstract}

Keywords: ethylal, 3,5-Dioxaheptane, thermochemistry, hot $\beta$ scission

\section{Introduction}

Oxygenated fuels and fuel additives are known for their ability to effectively reduce soot emissions of diesel engines [1] and are consequently studied with increasing interest. Moreover, most oxygenated fuels can be produced from biomass [2], which contributes to their popularity because oxygenated fuels may play a key role in the quest of reducing global carbon dioxide emissions. Nevertheless, despite their popularity, reaction kinetics research mainly focuses on smaller oxygenated molecules, while there are only few studies on larger oxygenated molecules available, such as i.e. poly(oxymethylene) dimethyl ethers [3]. In lack of reliable reaction kinetics data, analogies to other chemical groups are commonly used when modeling larger oxygenated fuels. However, a comprehensive ab initio kinetics prediction study on dimethoxymethane (DMM)

\footnotetext{
${ }^{*}$ Corresponding author:

Email address: leif .kroeger@ltt.rwth-aachen.de (Leif C. Kröger)
}

Preprint submitted to Proceedings of the Combustion Institute
[4] recently revealed that such kinds of analogies illdescribe the barely studied methylenedioxy group (O$\mathrm{CH}_{2}-\mathrm{O}$ ) [4]. The methylenedioxy group is also prominent in diethoxymethane (DEM) and the molecular structures of DMM and DEM are similar, but DEM contains $\mathrm{C}-\mathrm{C}$ bonds in opposite to DMM. These C-C bonds might in consequence influence the sooting tendency of DEM.

DEM itself can be produced on a large scale with gasto-liquid technology, which makes it a favorable potential diesel fuel additive [3]. Nevertheless, it is rarely studied in the context of combustion. H-abstraction by $\mathrm{OH}$ was studied experimentally at $298 \mathrm{~K}$ [5], 230372 K [6], 293-617 K [7], and abstraction by Oे was studied at $298 \mathrm{~K}$ [8]. Herzler et al. [9] investigated DEM decomposition in a single-pulse shock tube and observed 1.2-times and 0.5-times the amount of ethylene and ethanol for each consumed DEM, respectively. Fernández-Sánchez et al. [10] used oxygen-18 to perform DEM combustion experiments at $170{ }^{\circ} \mathrm{C}$ and dis- 
cussed the isotopic distribution of the tracer in the reaction products.

There exist two combustion mechanisms of DEM in the literature: Zhang et al. [3] were the first to report ignition delay time measurements of DEM and proposed a chemical kinetic mechanism describing the high-temperature oxidation of DEM. Dias et al. [11] derived a low-pressure kinetic model describing the effect of DEM addition to rich ethene flames and validated it against speciation data. In lack of sufficient data, both mechanisms have to rely mainly on analogies to describe most $\mathrm{H}$-abstraction and $\beta$-scission reactions. In addition, none of the mechanisms considers $\mathrm{H}$ abstraction by ethyl radicals despite the fact that ethyl radicals can be formed via $\beta$-scission of the fuel radicals.

It is consequently the aim of this study to provide high level $a b$ initio reaction rate constants for DEM Habstraction reactions by $\dot{\mathrm{H}}, \dot{\mathrm{C}} \mathrm{H}_{3}$, and $\dot{\mathrm{C}}_{2} \mathrm{H}_{5}$, and for the $\beta$-scission of DEM radicals. Thereby, a series of radicals relevant at pyrolysis condition is investigated. Also, these radicals correspond to beta-scission products and can therefore be of special importance to DEM chemistry. The relevance of the rarely studied $\mathrm{H}$-abstraction by ethyl radicals will be discussed and a first insight into the fate of the $\mathrm{C}-\mathrm{C}$ bonds will be given. Further, branching ratios for the so-called hot $\beta$-scission of rovibrationally excited fuel radicals are calculated to estimate the amount of excited radicals undergoing $\beta$-scission before being thermalized by collisions. Altogether, these rate constants will allow for more sophisticated and detailed future investigations of DEM and further elucidate the impact of the $\mathrm{O}-\mathrm{CH}_{2}-\mathrm{O}$ group in combustion kinetics.

\section{Methods}

Geometries and vibrational harmonic frequencies are obtained at B2PLYPD3BJ/6-311++(d,p) level throughout this study. Harmonic frequencies from B2PLYP level are known to outperform the widely accepted B3LYP level [12]; the same applies to bond lengths [13]. The geometries from the method mentioned above are used to calculate single point energies with CCSD(T) level of theory using extrapolation to the complete basis set (CBS) [14] according to

$$
E_{X}=E_{\mathrm{CBS}}+\frac{a}{X^{3}}
$$

with augmented double- and triple-zeta basis functions (aug-cc-pV(T+D)Z). The accuracy of this method for
$\mathrm{H}$-abstraction barriers has been benchmarked to 0.57 $\mathrm{kcal} / \mathrm{mol}$ [15]. T1-diagnostics [16] are used to check the adequateness of the single-reference approach. Gaussian09.d01 software was used for all electronic structure calculations (except DLPNO) calculations in this work [17].

The transition state structures of the $\mathrm{H}$-abstraction by ethyl radical reactions are found to be too large for CCSD(T)/aug-cc-pV(T+D)Z level of theory. Therefore, the recently developed DLPNO-CCSD(T) approximation $[18,19]$ is employed in these cases for the required single point calculations using the same basis sets and extrapolation to CBS recommended for DLPNO approximations [20], which is presented in the supporting information (SI). In order to benchmark this approach, all reaction barriers in this work (except barriers involving ethyl radicals) are calculated at both the $\operatorname{CCSD}(\mathrm{T})$ and the DLPNO-CCSD(T) (with TightPNO settings) level of theory. DLPNO-CCSD(T) calculations are performed via the ORCA program system (v. 4.0.1) [21]. The overall mean unsigned deviation (MUD) between the two methods of $0.127 \mathrm{kcal} / \mathrm{mol}$ (mean signed deviation $-0.074 \mathrm{kcal} / \mathrm{mol}$ ), while the maximum deviation amounts to $0.320 \mathrm{kcal} / \mathrm{mol}$. A detailed list of the energies and barrier heights can be found in the SI. These small deviations justify the application of the DLPNO method in the present system. Subsequently, the DLPNO reaction barriers are used in the scope of this work for predicting rate constants in the case of $\mathrm{H}$ abstraction by ethyl radicals.

Relaxed 1D rotor scans were performed using the B2PLYPD3BJ/6-311g++(d,p) method to ensure that the minimum energy conformer is found. If these scans revealed a new minimum, new subsequent $1 \mathrm{D}$ scans are started from this point again. Rate constants calculations are performed afterwards using the found minimum energy conformer and $1 \mathrm{D}$ hindered rotors with potential energy profiles obtained from the scans. Thereby, it is implied that all conformers can be reached from the minimum structures. We are aware that this is in general not guaranteed, however, we are confident that this procedure is sufficient in case of small molecules such as DEM. For each mode, torsional energy levels and the respective partition functions are obtained by solving the one-dimensional Schrödinger Equation using the python package TAMKin [22]. The reaction rate constants for hydrogen abstraction are calculated using conventional transition state theory (cTST) and the zero-curvature Eckart approach for tunnelling. Variational effects are expected to be of minor importance because of the rather high potential energy barriers and imaginary frequencies. The rate constants are calcu- 
lated from $500 \mathrm{~K}$ to $2000 \mathrm{~K}$ and the modified Arrhenius form is fitted to $\ln (k)$.

Temperature- and pressure-dependent rate constants for the unimolecular $\beta$-scission reactions are calculated via the Master Equation (ME) using the MESS software package [23]. The microcanonical rate constants are calculated from Rice-Ramsperger Kassel Marcus (RRKM) theory and tunneling is considered via Eckart potentials obtained from the well depths and imaginary frequencies of the transition states. The energy transfer via collisions is modeled via the weak collider bath gas argon using $\left\langle\Delta E_{\text {down }}\right\rangle=200 \mathrm{~cm}^{-1}(T / 300)^{0.85}$ while the Lennard-Jones collision frequency [24] is calculated from $\sigma=3.41$ and $6.65 \AA$ and $\epsilon=81.1$ and $351.0 \mathrm{~K}$ for argon and DEM, respectively. The Lennard-Jones collision parameters of $n$-heptane are used as close analog for DEM, while all other collision parameters are taken from Hippler et al. [25]. Again, modified Arrhenius parameters are fitted and reported for the calculated $k(T, p)$ at various pressures from 0.01 to $100 \mathrm{~atm}$.

Further, the concept of hot $\beta$-scission, which describes the dissociation of rovibrationally excited fuel radicals formed via hydrogen abstraction, is employed. Due to the excess energy available from hydrogen abstraction, the rovibrationally excited fuel radicals can dissociate faster than would be expected when assuming thermal equilibrium. Here, the rate constants calculated for hydrogen abstraction by $\dot{\mathrm{H}}, \dot{\mathrm{C}} \mathrm{H}_{3}$, and $\dot{\mathrm{C}}_{2} \mathrm{H}_{5}$ are used to quantify the fraction of DMM radicals undergoing hot $\beta$-scission. This is based on calculating the non-Boltzmann energy distribution of the DEM radicals, which in turn is used as input for ME simulations.

\section{Results and Discussion}

\subsection{Potential energy surface}

Three different fuel radicals can be formed by $\mathrm{H}$ abstraction: $\mathrm{R} 1\left(\mathrm{CH}_{2} \mathrm{CH}_{2} \mathrm{OCH}_{2} \mathrm{OCH}_{2} \mathrm{CH}_{3}\right)$ is formed by $\mathrm{H}$-abstraction from the outer methyl group, R2 $\left(\mathrm{CH}_{3} \mathrm{CHOCH}_{2} \mathrm{OCH}_{2} \mathrm{CH}_{3}\right)$ by abstraction of the adjacent $\mathrm{CH}_{2}$ group, and $\mathrm{R} 4\left(\mathrm{CH}_{3} \mathrm{CH}_{2} \mathrm{OC} \mathrm{HOCH}_{2} \mathrm{CH}_{3}\right)$ by abstraction from the central carbon atom. The radical $\mathrm{R} 2$ is the most stable out of these three and it has also the lowest barrier for formation by $\mathrm{H}$-abstraction (s. PESs in Fig. 1). The barriers presented if Fig. 1 refer to $\mathrm{H}$-abstraction by $\dot{\mathrm{H}}$, while the barriers for $\mathrm{H}$-abstraction by $\dot{\mathrm{CH}}_{3}$ and $\dot{\mathrm{C}}_{2} \mathrm{H}_{5}$ lie roughly $3 \mathrm{kcal} / \mathrm{mol}$ higher. Energetic differences between $\mathrm{R} 2$ and $\mathrm{R} 4$ are small for both the stable structures and the transition state structures, while the barrier is significantly higher in case of R1. Despite these close energetic similarities of R2 and
$\mathrm{R} 4$, they are connected via a large isomerization barrier (even higher than the respective $\beta$-scission barriers) indicating that such an isomerization will be of no practical relevance. The isomerization barriers for forming $\mathrm{R} 2$ or $\mathrm{R} 4$ from $\mathrm{R} 1$ are smaller than the $\beta$-scission barrier of $\mathrm{R} 1$, making $\beta$-scission of $\mathrm{R} 1$ unlikely. $\mathrm{R} 1$ will consequently either isomerize to one of the other radicals or undergo $\mathrm{O}_{2}$-addition. The difference of the barriers for isomerization to $\mathrm{R} 1$ and $\beta$-scission deviate less than $3 \mathrm{kcal} / \mathrm{mol}$ in case of R 2 causing isomerization, $\beta$-scission, and $\mathrm{O}_{2}$ addition to be competing pathways here. $\mathrm{R} 4$ possesses the smallest $\beta$-scission barrier, which is significantly smaller than the isomerization barriers. This suggests that once R4 is formed it will either undergo $\mathrm{O}_{2}$ addition or $\beta$-scission. Please note that $\mathrm{O}_{2}$ addition is not investigated in this study and that branching ratios between $\beta$-scission and $\mathrm{O}_{2}$ addition can only be estimated from kinetic simulations. Since the $\beta$-scission barrier of R4 is of similar height as the reverse hydrogen barrier, it might be possible that freshly formed R4 radicals have enough excess energy to undergo $\beta$-scission directly (so-called hot $\beta$-scission [26]) without being thermalized.

When analyzing the $\beta$-scission products, ethylene and the ethyl radical stand out. Ethylene can be considered as a soot precursor [27] and is formed by $\beta$-scission of $\mathrm{R} 1$. However, it will be formed rarely due to the low significance of the $\mathrm{R} 1 \beta$-scission pathway and thus, will not contribute to soot formation. Ethyl radicals originate from $\mathrm{R} 4 \beta$-scission and from subsequent $\beta$-scission of the R2- $\beta$-scission product $\dot{\mathrm{CH}}_{2} \mathrm{OCH}_{2} \mathrm{CH}_{3}$. Because $\beta$-scission of $\mathrm{R} 4$ is a predominant pathway, especially under fuel rich or pyrolysis-like conditions, one expects significant ethyl radical production, which then contribute to $\mathrm{H}$-abstraction.

\subsection{Thermochemistry}

Enthalpies, entropies, and heat capacities were calculated for DEM and its radicals to be used in detailed combustion models. The results are reported in the SI along with fitted NASA-polynomial expressions. The standard enthalpy of formation of DEM is calculated via the isodesmic reaction DEM $+\mathrm{C}_{2} \mathrm{H}_{6}$ $\leftrightarrow \mathrm{DMM}+\mathrm{C}_{4} \mathrm{H}_{10}$ to $\Delta h_{\mathrm{f}}(298.15 \mathrm{~K})=-99.28 \mathrm{kcal} / \mathrm{mol}$, which agrees within $0.2 \mathrm{kcal} / \mathrm{mol}$ with the experimental value of $\Delta h_{\mathrm{f}}^{\text {exp. }}(298.15 \mathrm{~K})=-99.14 \mathrm{kcal} / \mathrm{mol}$ [28]. Please refer to the SI for details and experimental values used [29].

\subsection{H-atom abstraction}

Three-parameter Arrhenius expressions are fitted to reaction rate constants for DEM $\mathrm{H}$-abstraction by $\dot{\mathrm{H}}$, 


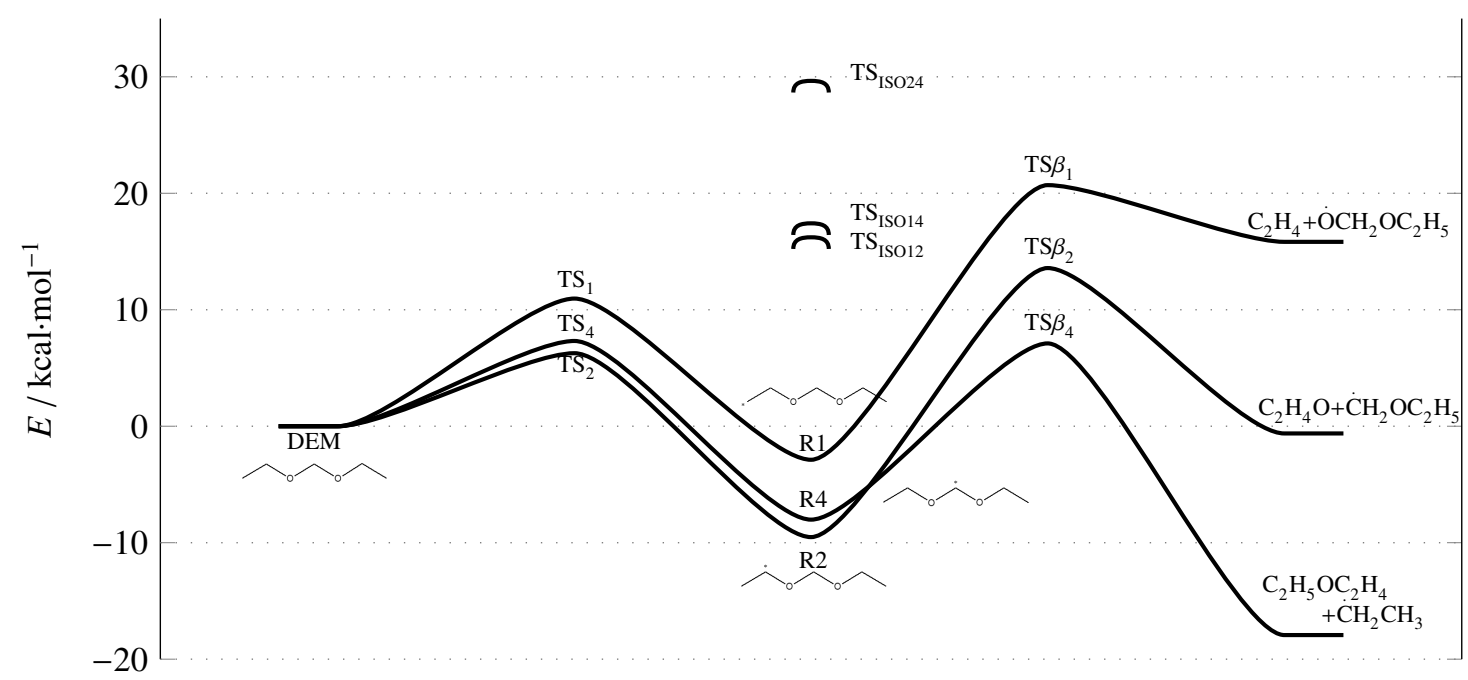

Figure 1: Potential energy surfaces of DEM H-abstraction, radical isomerization, and radical $\beta$-scission. The energy of DEM $+\dot{\mathrm{H}}$ is set to zero and energetic differences due to different stoichiometric formulas are accounted for by subtracting the energies of $\mathrm{H}_{2}$. $\mathrm{H}$-abstraction barriers shown here denote $\mathrm{H}$-abstraction by $\dot{\mathrm{H}}$.

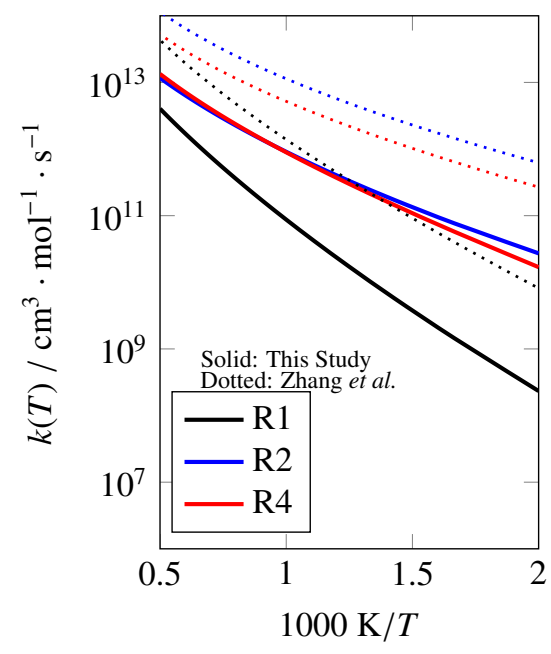

(a) $\mathrm{RH}+\dot{\mathrm{H}} \longrightarrow \mathrm{R}+\mathrm{H}_{2}$

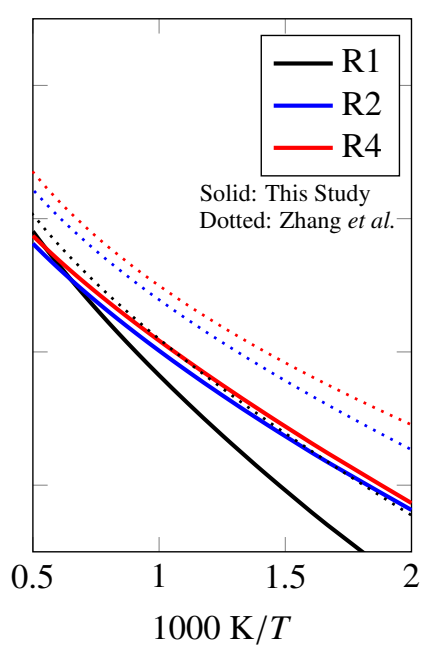

(b) $\mathrm{RH}+\dot{\mathrm{C}} \mathrm{H}_{3} \longrightarrow \mathrm{R}+\mathrm{CH}_{4}$

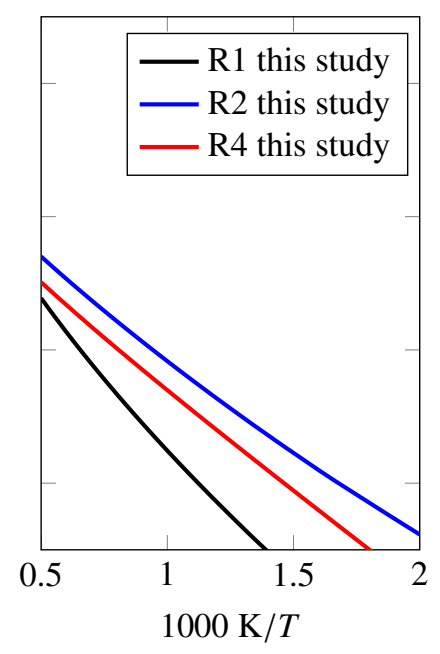

(c) $\mathrm{RH}+\dot{\mathrm{C}}_{2} \mathrm{H}_{5} \longrightarrow \mathrm{R}+\mathrm{C}_{2} \mathrm{H}_{6}$

Figure 2: Site-specific rate constants for $\mathrm{H}$-abstraction from DEM. The solid lines are the ones computed in this study and the dotted lines denote rate constants from Zhang et al. [3]. Barriers used in cTST calculations in case of $\dot{\mathrm{C}}_{2} \mathrm{H}_{5}$ are obtained from CCSD(T)-DLPNO calculations (cf. method section).

$\dot{\mathrm{C}} \mathrm{H}_{3}$, and $\mathrm{C}_{2} \mathrm{H}_{5}$ calculated and presented in Tab. 1. Tunneling increases the rate constants by a factor up to 2 at $500 \mathrm{~K}$ and the influence decreases with rising temperature. Hindered rotor contributions effect the rate constants by a factor of up to 3 .

All rates are plotted in Fig. 2 over the calculated temperature range alongside with values used in the modeling study of Zhang et al. [3]. At first, it can be seen clearly that abstraction from R2 and R4 is taking place at com- parable rate constants for all involved radicals and is always faster than abstraction from R1. Further, it can be observed that $\mathrm{H}$-abstraction by $\dot{\mathrm{H}}$ results in the largest rate constants. Both observations are in good agreement with the conclusions drawn from the PESs presented before. Under some conditions abstraction at R4 site is faster compared to the R2 site, which results from differing reaction entropies.

The comparison of the rate constants calculated in this 
Table 1: Modified Arrhenius equation ${ }^{\dagger}$ parameters of the temperaturedependent hydrogen abstraction rate constants for abstraction from DEM via $\dot{\mathrm{H}}, \dot{\mathrm{C}} \mathrm{H}_{3}$ and, $\dot{\mathrm{C}}_{2} \mathrm{H}_{5}$. The temperature limit is $500 \mathrm{~K}-2000 \mathrm{~K}$ for all reactions and indicates the validity ranges for the parameters.

\begin{tabular}{lcrr} 
Reaction & $A / \frac{\mathrm{cm}^{3}}{\mathrm{~mol} \mathrm{~s}}$ & $n /-$ & $E_{\mathrm{a}} / \frac{\mathrm{cal}}{\mathrm{mol}}$ \\
\hline \hline $\mathrm{RH}+\dot{\mathrm{H}} \longrightarrow \mathrm{R} 1+\mathrm{H}_{2}$ & $1.403 \cdot 10^{+5}$ & 2.537 & 8320.8 \\
\hline $\mathrm{RH}+\dot{\mathrm{H}} \longrightarrow \mathrm{R} 2+\mathrm{H}_{2}$ & $6.858 \cdot 10^{+5}$ & 2.318 & 3810.8 \\
\hline $\mathrm{RH}+\dot{\mathrm{H}} \longrightarrow \mathrm{R} 4+\mathrm{H}_{2}$ & $6.078 \cdot 10^{+6}$ & 2.087 & 5009.2 \\
\hline $\mathrm{RH}+\dot{\mathrm{C}} \mathrm{H}_{3} \longrightarrow \mathrm{R} 1+\mathrm{CH}_{4}$ & $3.732 \cdot 10^{-1}$ & 3.727 & 9637.9 \\
\hline $\mathrm{RH}+\dot{\mathrm{C}} \mathrm{H}_{3} \longrightarrow \mathrm{R} 2+\mathrm{CH}_{4}$ & $1.898 \cdot 10^{+2}$ & 2.769 & 7175.6 \\
\hline $\mathrm{RH}+\dot{\mathrm{C}} \mathrm{H}_{3} \longrightarrow \mathrm{R} 4+\mathrm{CH}_{4}$ & $6.200 \cdot 10^{+3}$ & 2.369 & 7916.2 \\
\hline $\mathrm{RH}+\dot{\mathrm{C}}_{2} \mathrm{H}_{5} \longrightarrow \mathrm{R} 1+\mathrm{C}_{2} \mathrm{H}_{6}$ & $9.721 \cdot 10^{-2}$ & 3.641 & 11096.3 \\
\hline $\mathrm{RH}+\dot{\mathrm{C}}_{2} \mathrm{H}_{5} \longrightarrow \mathrm{R} 2+\mathrm{C}_{2} \mathrm{H}_{6}$ & $2.333 \cdot 10^{+5}$ & 1.842 & 9451.1 \\
\hline $\mathrm{RH}+\dot{\mathrm{C}}_{2} \mathrm{H}_{5} \longrightarrow \mathrm{R} 4+\mathrm{C}_{2} \mathrm{H}_{6}$ & $3.304 \cdot 10^{+8}$ & 0.865 & 12430.6 \\
\hline
\end{tabular}

${ }^{\dagger}$ Modified Arrhenius equation: $k(T)=A T^{n} \exp \left(-E_{\mathrm{a}} / R / T\right)$

work to the values used by Zhang et al. [3] reveals that despite showing the same trend, the rate constants deviate by up to 2 orders of magnitude. Zhang et al. [3] use analogies to diethylether for the abstractions leading to R1 and R2, and they use analogies to DMM (the rate constants of which were obtained from analogies, as well) for the central abstraction site (leading to R4). These rather large deviations are in agreement with the observation made by Kopp et al. [4] who concluded for DMM that rate constants obtained from analogies appear to ill-describe the rarely studied methylenedioxy group and in turn contribute to large deviations. In fact, comparing the rate constants of this study to the rate constants of DMM [4] reveals that the rate constants of the DEM-R4 site correspond well to the DMM-R3 (central atom). As expected, the DEM-R1 does not compare well to the DMM-R1, which is due to the latter having one neighboring oxygen and the former having one additional neighboring carbon. This indicates that rate constant analogies work well for abstraction from the central carbon atom only, if both molecules contain the methylenedioxy group.

Both available kinetic modeling studies do not consider $\mathrm{H}$-abstraction by ethyl radicals. The results of this work clearly show that rate constants for $\mathrm{H}$-abstraction by ethyl radicals are similar to those for $\mathrm{H}$-abstraction by methyl radicals. Together with fact that ethyl-radicals are the predominant $\beta$-scission product, we propose that abstraction by ethyl radicals should be considered in DEM modeling studies. In fact, the previous overestimation of the rate constants for $\mathrm{H}$-abstraction by methyl radicals might origin from neglecting the ethyl $\mathrm{H}$-abstraction reactions.

\subsection{Thermal unimolecular radical kinetics}

Isomerization between the three DEM radicals is of minor importance for most conditions. While R2 and R4 practically never isomerize to any other radical, $\mathrm{R} 1$ predominantly isomerizes at high-pressure and lowtemperature conditions. With increasing temperature and decreasing pressure, however, isomerization of R1 becomes less important. It is noteworthy, however, that at low pressure isomerization of $\mathrm{R} 1$ to $\mathrm{R} 2$ is about ten times faster compared to isomerization of R1 to R4. At high-pressure, this isomerization branching is reversed, i.e. isomerization of R1 to R4 is ten times faster compared to isomerization to $\mathrm{R} 2$.

The $\beta$-scission rate constants of all radicals show a strong temperature- and pressure-dependent fall-of. The $T, p$-dependent fall-off behavior of all three radicals is very similar, which is why only the results for R4 is shown in SI. The fall-off is getting stronger with increasing temperature and decreasing pressure, which is expected. This effect, however, is more pronounced than for DMM radicals [4].

Figure 3 illustrates that R4 is associated with the largest $\beta$-scission rate constants and $\mathrm{R} 1$ with the smallest, which confirms earlier observations from the PES. At low temperatures the difference exceeds two orders of magnitude. In addition, even at high pressures of $100 \mathrm{~atm}$, the $\beta$-scission rate constants have still not reached the high pressure limit. This is especially true at high temperatures (cf. Fig. 3), which clearly indicates that the pressure-dependence of these reaction needs to be considered when modeling DEM combustion at engine relevant conditions. Rate constants are provided in the SI.

\subsection{Hot unimolecular radical kinetics}

Döntgen et al. [26] studied the possibility of "prompt" radical beta-scission of radicals formed via hydrogen abstraction, the so-called hot $\beta$-scission. Radicals may possess enough excess energy immediately after formation, so that they dissociate before being thermalized. Earlier, it was discussed based on the PESs that especially radical R4 might be prone to hot $\beta$-scission, because the $\beta$-scission barrier is of similar height as the reverse hydrogen abstraction barrier. Therefore, the temperature- and pressure-depending ratios for hot $\beta$-scission of $\mathrm{R} 4$ formed via $\mathrm{H}$-abstraction by $\dot{\mathrm{C}} \mathrm{H}_{3}$ are presented in Fig. 4 . The hot $\beta$-scission branching ratios of $\mathrm{R} 4$ formed by the $\dot{\mathrm{H}}$ or $\mathrm{CH}_{2} \mathrm{CH}_{3}$ have a similar behavior. Hot $\beta$-scission turns out be relevant at very low pressures and moderate to high temperatures only. Even at $1 \mathrm{~atm}$ and $1000 \mathrm{~K}$ hot $\beta$-scission has 


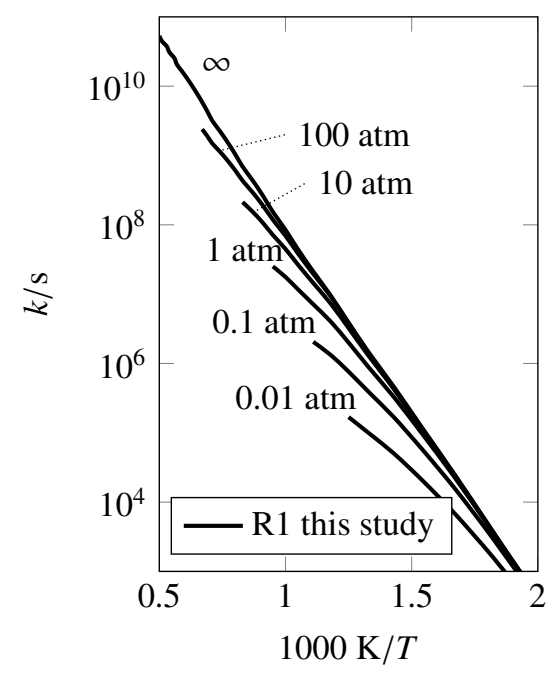

(a) $\mathrm{R} 1 \beta$-scission

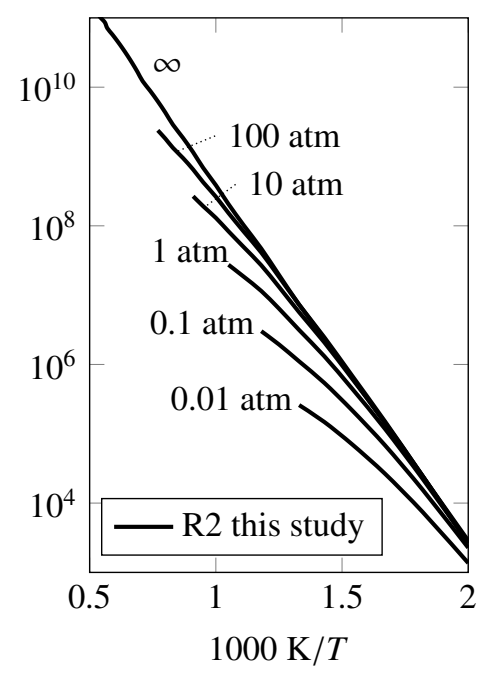

(b) R2 $\beta$-scission

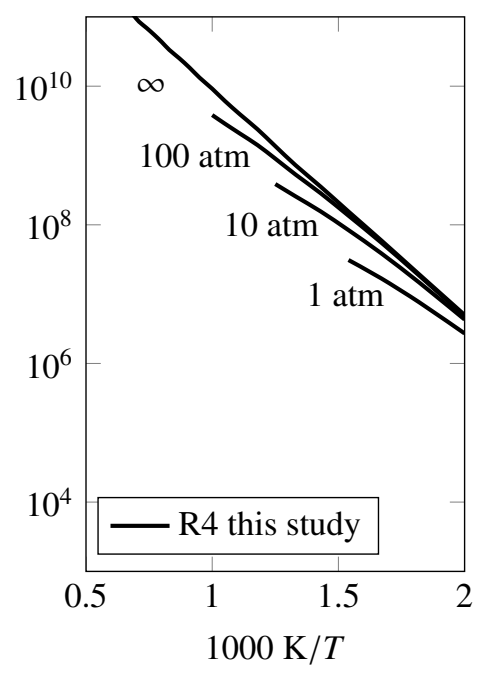

(c) R4 $\beta$-scission

Figure 3: Temperature- and pressure-depending rate constants for thermal $\beta$-scission of R1, R2, and R4 (solid lines).

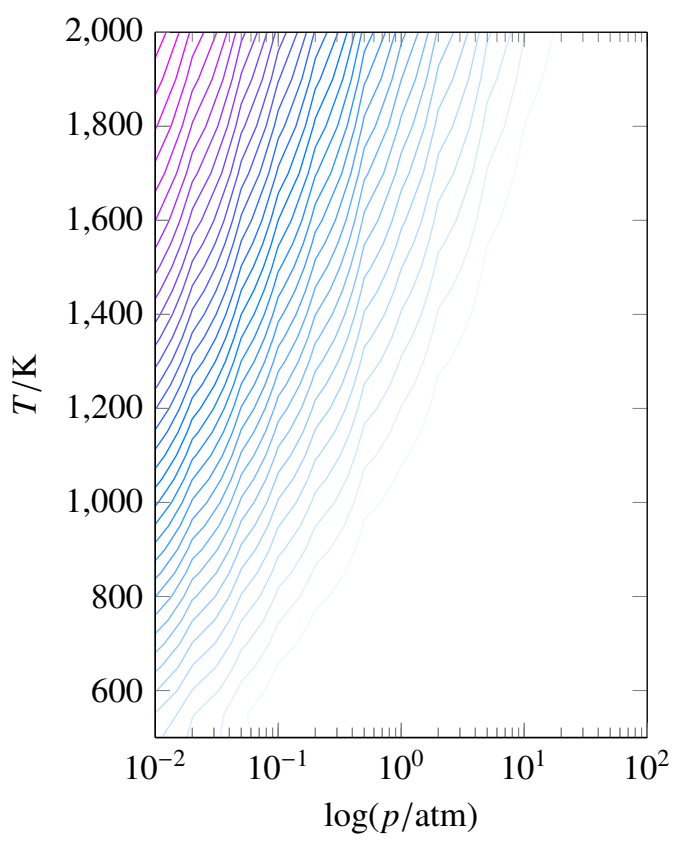

Figure 4: Temperature- and pressure-depending branching ratios for hot $\beta$-scission of the R4 formed via $\mathrm{H}$-abstraction by $\dot{\mathrm{C}} \mathrm{H}_{3}$.

barely an effect, but at 1 bar and $1500 \mathrm{~K}$ it accounts for roughly $20 \%$ of the $\beta$-scission reactions. Consequently, hot $\beta$-scission can be neglected at engine relevant conditions, but might be relevant when studying flames at atmospheric conditions.

\subsection{Conclusions}

0.6

The present work represents the first high level $a b$ 0.5 initio study of DEM and provides valuable thermochemistry data as well as reaction kinetic data for $\mathrm{H}$ abstraction by $\dot{\mathrm{H}}, \dot{\mathrm{C}} \mathrm{H}_{3}$, and $\dot{\mathrm{C}} \mathrm{H}_{2} \mathrm{CH}_{3}$ and for subsequent unimolecular reaction. $\mathrm{H}$-abstraction mainly leads to $\mathrm{R} 2$ and R4 formation at almost similar rates. It could

0.3 be demonstrated that $\mathrm{H}$-abstraction by ethyl radicals denotes a reasonable pathway due to the availability of ethyl radicals from DEM radical $\beta$-scission and high rate constants. This pathway should be investigated further by kinetic modeling studies. Further, $\beta$-scission

0.1 effects predominantly $\mathrm{R} 4$ and reveals that $\beta$-scission pathways leading to formation of ethylene are of minor importance, which indicates that the formation of low amounts of ethylene might be a reason for the lower sooting tendency of DEM compared to other $\mathrm{C}-\mathrm{C}$ bond containing species. The hot $\beta$-scission concept was applied and found to mainly effect the R4 radical at high temperatures and low pressures. In addition, it was once more observed that analogies to other chemical groups seem to work poorly in case of the methylenedioxy group. Analogies to compounds containing a methylenedioxy group, however, pose adequate analogies (such as DMM). The presented insights into the kinetics and thermochemistry of DEM and its radicals enable more accurate and comprehensive kinetic modeling of DEM. 


\section{Acknowledgments}

This work was performed as part of the Cluster of Excellence "Tailor-Made Fuels from Biomass" (EXC 236), which is funded by the Excellence Initiative by the German federal and state governments to promote science and research at German universities. We gratefully acknowledge CPU time grant rwth0070 of the RWTH Aachen University Compute Cluster. DF is thankful for financial support from the Deutsche Forschungsgemeinschaft (German Research Association) through grant GSC 111.

\section{Supplementary material}

Geometries, thermochemistry data, unimolecular rate constants, $\operatorname{CCSD}(\mathrm{T}) /$ aug-cc-pV $(\mathrm{T}+\mathrm{D}) \mathrm{Z}$ energies, DLPNO-CCSD(T)/aug-cc-pV(T+D)Z energies, and reaction barriers obtained form both energy methods are reported in the supporting information.

\section{References}

[1] L. S. Tran, B. Sirjean, P.-A. Glaude, R. Fournet, F. BattinLeclerc, Energy 43 (1) (2012) $4-18$.

[2] F. Agblevor, S. Besler, A. Wiselogel, Fuel Sci. Technol. Int. 14 (4) (1996) 589-612.

[3] C. Zhang, J. He, Y. Li, X. Li, P. Li, Fuel 154 (2015) 346 - 351.

[4] W. A. Kopp, L. C. Kröger, M. Döntgen, U. Burke, H. J. Curran, K. A. Heufer, K. Leonhard, Combust. Flame accepted for publication.

[5] P. Dagaut, R. Liu, T. J. Wallington, M. J. Kurylo, Int. J. Chem. Kinet. 21 (12) (1989) 1173-1180.

[6] E. Porter, J. Wenger, J. Treacy, H. Sidebottom, A. Mellouki, S. Téton, G. LeBras, J. Phys. Chem. A 101 (32) (1997) 57705775.

[7] C. Vovelle, A. Bonard, V. Daële, J.-L. Delfau, Phys. Chem. Chem. Phys. 3 (22) (2001) 4939-4945.

[8] R. Liu, P. Dagaut, R. E. Huie, M. J. Kurylo, Int. J. Chem. Kinet. 22 (7) (1990) 711-717.

[9] J. Herzler, J. A. Manion, W. Tsang, J. Phys. Chem. A 101 (30) (1997) 5494-5499.

[10] E. Fernández-Sánchez, J. Garría-Domínguez, M. Molera, J. Santiuste, Z. f. Phys. Chem. 270 (1) (1989) 289-296.

[11] V. Dias, J. Vandooren, Combust. Flame 158 (5) (2011) 848 859.

[12] M. Biczysko, P. Panek, G. Scalmani, J. Bloino, V. Barone, J. Chem. Theory Comput. 6 (2010) 2115-2125.

[13] F. Neese, T. Schwabe, S. Grimme, J. Chem. Phys. 126 (12) (2007) 124115.

[14] K. L. Bak, A. Halkier, P. Jørgensen, J. Olsen, T. Helgaker, W. Klopper, J. Mol. Struct. 567 (2001) 375-384.

[15] J. Zheng, Y. Zhao, D. G. Truhlar, J. Chem. Theory Comput. 5 (4) (2009) 808-821.

[16] T. J. Lee, P. R. Taylor, Int. J. Quantum Chem. 23 (1989) 199207.

[17] M. J. Frisch et. al., Gaussian 09 (2009).

[18] C. Riplinger, B. Sandhoefer, A. Hansen, F. Neese, The Journal of Chemical Physics 139 (13) (2013) 134101.
[19] C. Riplinger, F. Neese, J. Chem. Phys. 138 (3) (2013) 034106.

[20] D. G. Liakos, F. Neese, J. Phys. Chem. A 116 (19) (2012) 48014816.

[21] F. Neese, WIREs Comput. Mol. Sci. 2 (1) (2012) 73-78

[22] A. Ghysels, T. Verstraelen, K. Hemelsoet, M. Waroquier, V. Van Speybroeck, J. Chem. Inf. Model. 50 (2010) 1736-1750.

[23] Y. Georgievskii, J. A. Miller, M. P. Burke, S. J. Klippenstein, J. Phys. Chem. A 117 (2013) 12146.

[24] H.-H. Carstensen, A. M. Dean, Compr. Chem. Kinet. 42 (2007) 105-187.

[25] H. Hippler, J. Troe, H. J. Wendelken, J. Chem. Phys. 78 (1983) 6709.

[26] M. Döntgen, L. C. Kröger, K. Leonhard, Proc. Combust. Inst. 36 (2017) 135-142.

[27] G. Blanquart, P. Pepiot-Desjardins, H. Pitsch, Combust. Flame 156 (2009) 588-607.

[28] M. Månsson, J. Chem. Thermodyn. 1 (2) (1969) 141 - 151.

[29] D. A. Pittam, G. Pilcher, J. Chem. Soc., Faraday Trans. 168 (1972) 2224-2229. 